\title{
Astrometry of water masers in post-AGB stars
}

\author{
Hiroshi Imai $^{1}$ and VERA collaboration ${ }^{1,2}$ \\ ${ }^{1}$ Department of Physics and Astronomy, Graduate School of Science and Engineering, \\ Kagoshima University, 1-21-35 Korimoto, Kagoshima 890-0065, Japan \\ email: hiroimai@sci.kagoshima-u.ac.jp \\ ${ }^{2}$ Mizusawa VLBI Observatory, National Astronomical Observatory of Japan, \\ 2-12 Hoshigaoka, Mizusawa-Ku, Oshu-shi, Iwate 023-0861, Japan
}

\begin{abstract}
Measurements of trigonometric parallaxes and secular motions of evolved stars, especially post-AGB stars including central objects of planetary nebulae and water fountain sources as well as peculiar or unclassified stars, provide unambiguous source distance scales and information on their orbits in the Milky Way Galaxy. True source luminosities and kinematical properties should lead us to elucidate the true characteristics and evolutional tracks of these stars. Here we present the recent results of astrometry towards $\mathrm{H}_{2} \mathrm{O}$ maser sources with the VLBI Exploration of Radio Astormetry (VERA). The target sources include a planetary nebula (K3-35), a pre-PN (IRAS 19312+1950), a water fountain (IRAS 18286-0959) and a K-type star (IRAS 22480+6002). We have demonstrated that parental stars of the former three sources should be intermediate-mass stars from their luminosities and orbits in the Milky Way. It is suggested that IRAS $22480+6002$ should be a K-type supergiant previously suggested rather than an RV Tau variable star.
\end{abstract}

Keywords. masers, stars:distances, individuals (K3-35, IRAS 19312+1950, IRAS 18286-0959, IRAS $22480+6002)$

The dual-beam receiver system of the VERA telescopes enables us to simultaneously track a Galactic $\mathrm{H}_{2} \mathrm{O}$ maser source and an position-reference quasar, yielding submilliarcsecond-level astrometry between the two sources separated by $0.3^{\circ}-2.2^{\circ}$. In spite of high time variability of the $\mathrm{H}_{2} \mathrm{O}$ masers, a few maser spots survive for one year or longer, enabling us to monitor their motions to measure their trigonometric parallaxes and linear proper motions.

We have conducted astrometric VLBI observations of $\mathrm{H}_{2} \mathrm{O}$ masers in four post-AGB stars with VERA. Some of the results were published by Imai et al. (2011) and Tafoya et al. (2011)). Fig. 1 shows one of the results of the astrometric observations. Table 1 gives astrometric and Galactic kinematical parameters of these sources derived from the astrometric results. These sources harbor bipolar morphology of PNe or "water fountains" (WFs). From the VERA astrometry, we have learnt the following.

1. Stellar luminosities derived from the spectral energy distributions and the trigonometric parallax distances are higher than $10000 L_{\odot}$ for IRAS 18286-0959 and IRAS $19312+1950$ and $35000 L_{\odot}$ for IRAS $22480+6002$. Taking into account the size of the $\mathrm{H}_{2} \mathrm{O}$ maser distribution ( $>100 \mathrm{AU}$ ) as well, IRAS $22480+6002$ should be a K-type supergiant rather than an RV Tau variable star.

2. Large deviations from the Galactic circular rotation, $>50 \mathrm{~km} \mathrm{~s}^{-1}$ for IRAS $18286-0959$, implies kinematical property different from that of massive young stellar objects in the Galactic thin disk. These imply that the central stars may be intermediate-mass post AGB stars. Our recent sub-mm CO line observations also support this mass estimation (c.f. IRAS 16342-3814, Imai et al. 2012). 
Table 1. Source parameters derived from the VERA astrometry.

\begin{tabular}{|c|c|c|c|c|c|c|c|c|}
\hline Object & Type & $\begin{array}{r}D \\
(\mathrm{kpc})\end{array}$ & $\begin{array}{r}R_{\mathrm{Gal}} \dagger \\
(\mathrm{kpc})\end{array}$ & $\begin{array}{r}z \ddagger \\
(\mathrm{pc})\end{array}$ & $\begin{array}{c}V_{R} \\
\left(\mathrm{~km} \mathrm{~s}^{-1}\right)\end{array}$ & $\begin{array}{r}V_{\theta} \text { ฯ } \\
\left(\mathrm{km} \mathrm{s}^{-1}\right)\end{array}$ & $\begin{array}{c}V_{z} \\
\left(\mathrm{~km} \mathrm{~s}^{-1}\right)\end{array}$ & Reference \\
\hline $\mathrm{K} 3-35$ & $\mathrm{PN}$ & $3.9_{-0.5}^{+0.7}$ & $7.11_{-0.06}^{+0.08}$ & $140_{-18}^{+25}$ & $33 \pm 16$ & $233 \pm 11$ & $11 \pm 2$ & Tafoya et al. (2011) \\
\hline IRAS $19312+1950$ & Pre-PN & $3.8_{-0.6}^{+0.8}$ & $7.07 \pm 0.12$ & $28 \pm 3$ & $33 \pm 28$ & $214 \pm 4$ & $-14 \pm 8$ & Imai et al. (2011) \\
\hline IRAS $18286-0959$ & WF & $3.9_{-0.7}^{+1.1}$ & $4.93 \pm 0.69$ & $22 \pm 2$ & $64 \pm 30$ & $133 \pm 36$ & $-17 \pm 31$ & Imai et al. in prep. \\
\hline IRAS $22480+6002$ & K-type & $2.50_{-0.23}^{+0.28}$ & $9.10 \pm 0.14$ & $55 \pm 4$ & $203 \pm 5$ & $55 \pm 4$ & $4 \pm 1$ & Imai et al. in prep. \\
\hline
\end{tabular}

$\dagger$ Galactocentric distance to the object. $\ddagger$ Height from the Galactic midplane.

ฯ $V_{R}, V_{\theta}$, and $V_{z}$ are the secular motion vector components in the Galactic radial, azimuthal, and rotation axis directions, respectively.
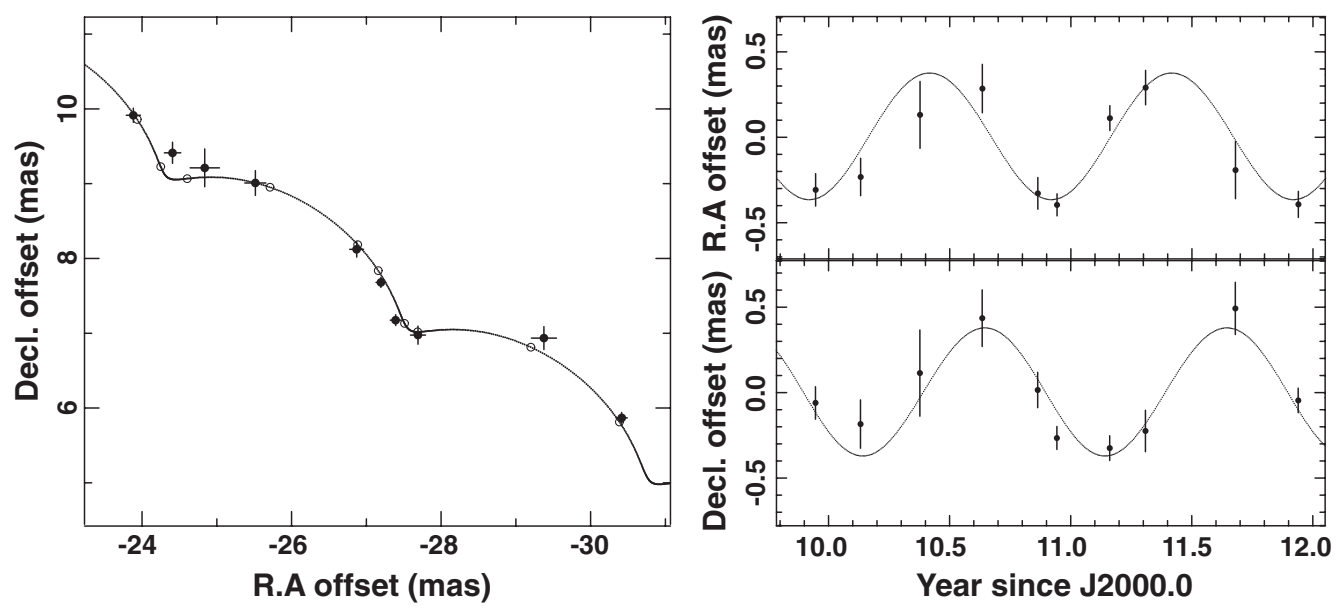

Figure 1. $\mathrm{H}_{2} \mathrm{O}$ maser spot position in IRAS $22480+6002$ and the kinematical model for the spot's motion. Left R.A. and decl. offsets of the maser spot observed (a filled circle) and model-predicted (an opened circle). A solid curve shows the modeled motion including an annual parallax and a constant velocity proper motion. Right R.A. and decl. variations of the spot position with time. The estimated linear proper motion is subtracted from the observed spot position.

3. Accuracy of kinematical parameters of the maser sources depend on accuracy of the relative motion of the position reference maser feature, whose annual parallax and secular motion is measured, with respect to the systemic motion of the maser source $\left(\sigma \sim 10 \mathrm{~km} \mathrm{~s}^{-1}\right)$, except for water fountain sources whose jet velocities are higher than several kilometers per second.

\section{References}

Imai, H., Chong, S.-N., He, J.-H., Nakashima, J., Hsia, C.-H., Sakai, T., Deguchi, S., \& Koning, N. 2012, PASJ, submitted

Imai, H., Tafoya, D., Honma, M., Hirota, T., \& Miyaji, T. 2011, PASJ, 63, 87

Tafoya, D., Imai, H., Gómez, Y. Torrelles, J. M., Patel, N. A., Anglada, G., Miranda, L. F., Honma M., Hirota, T., \& Miyaji, T. 2011, PASJ, 63, 71 